\title{
Electrochemical-Coupled Vibrational Spectromicroscopy Recognize Conductive Islands in Topological Redox Surface
}

Ayaz Hassan, Lucyano J. A Macedo and Frank N. Crespilho*

São Carlos Institute of Chemistry, University of São Paulo, São Paulo, 13560-970, Brazil

\begin{abstract}
We introduce a set up to observe real time changes in the electronic states of polymeric islands by monitoring the redox states of polyaniline using electrochemicalcoupled vibrational spectromicroscopy (EVSM). Unique 2D and 3D chemical maps reveal electrochemical heterogeneity, showing promising topological properties controls.
\end{abstract}

Introduction

In flexible electronics and bioelectronics, it is still a great challenge to get an unambiguous correlation between the topological structural order and electrical conductivity. For instance, polyaniline (PAni) thin films and its composites have useful applications in flexible electronics and have been revolutionized the actual generation of high-tech devices, such as molecular imprinted polymer based chemical sensor ${ }^{1}$, organic electronics ${ }^{2}$ and communication devices ${ }^{3}$. Generally, Atomic Force Microscopy (AFM)-based techniques are used to reveal the out-of-plane elastic moduli and charge mobilities ${ }^{4}$, which is important to obtain conductive films with ordered topology. However, AFM requires long times and has poor spatial resolution in localizing real-time topological distortion within larger areas of the electrodes. Here we introduce an Electrochemical-Coupled Vibrational Spectromicroscopy (EVSM) technique, ${ }^{5}$ that is about 100 times faster than AFM, with the possibility of analysing large surfaces in few minutes, and with the advantage of obtaining both spectroscopic and electronic information of the conductive surfaces with micrometer range spatial resolution. EVSM technique was applied in this work under two different perspective: i) PAni thin film as a redox polymer and showing real time changes on the surface morphology as a function of applied electrochemical potential despite of the spectral interferences from the absorption modes of the water molecules in the studied region and ii) PAni as a conducting polymer and showing the topological structural changes in term of optical microscopic and spectromicroscopic chemical images. EVSM is introduced here as a 


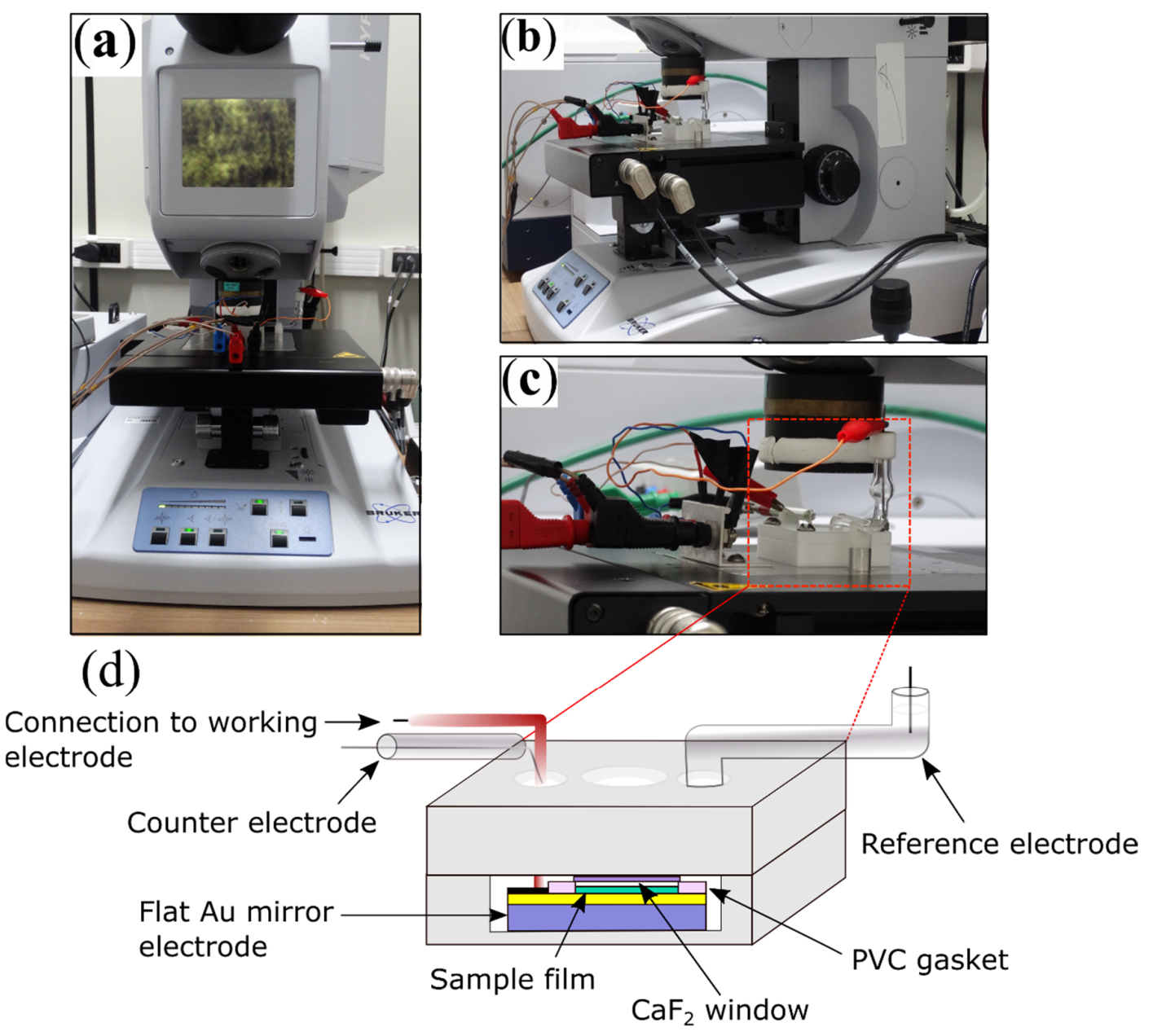

Fig. 1. EVSM instrumental set up. Photographs of the FTIR microscope coupled to electrochemistry through a home-built electrochemical cell mounted to the microscope stage (a) frontal view (b) lateral view (c) microscope objective and spectroelectrochemical cell (d) sketch of the of the cell exhibiting the connections of the reference and counter electrodes (top) and the position of working electrode, PVC, electrolyte, and $\mathrm{CaF}_{2}$ window (bottom).

powerful technique to explore topological effects. Briefly, EVSM comprise a multiplex FTIR chemical imaging from focal plane array (FPA) detector connected to an inverted electrochemical cell, as shown in Fig.1. Specific vibrational modes are selected using FPA, allowing the collection of 4096 IR spectra from the scanned area of the sample (micro-FTIR) in a single detection, which then allows the construction of the chemical maps showing the surface changes in colour patterns, providing useful information regarding the charge transfer processes and its impact on the chemical composition of 
the probed material in specific spatially resolved 2D regions ${ }^{6}$. A home-made three electrodes spectroelectrochemical cell containing the PAni modified flat Au mirror as the working electrode, $\mathrm{Pt}$ wire sealed in a glass tube as counter electrode and $\mathrm{Ag} / \mathrm{AgCl}$ sat as a reference electrode (see Fig. 1d) is used for EVSM analysis. The spectroelectrochemical cell was positioned in the motorized stage of the FTIR microscope coupled to an FTIR spectrometer, which provides the facility of moving the electrochemical cell and hence the measurement can be performed at any desired position of the working electrode by initially focusing the visible and then IR light through $\mathrm{CaF}_{2}$ window when the measurement is performed (see Fig 1a-c). The $\mathrm{CaF}_{2}$ window also controls the thickness of the electrolyte layer above the working electrode. A gasket made of PVC was placed between the $\mathrm{CaF}_{2}$ window and the surface of the working electrode (Fig. 1d). The working electrode was obtained by electrodepositing the PAni on flat Au mirror through the application of fixed potential (i.e $0.8 \mathrm{~V}$ ) in a polymerization solution containing freshly distilled aniline and $\mathrm{H}_{2} \mathrm{SO}_{4}$ followed by cyclic voltammetry experiments, obtaining the characteristics redox couples (Fig. 2a-b), which are consistent to the interconversion of different forms of the PAni. Prior to performing the EVSM, the PAni electrode was characterized through micro-FTIR (for experimental detail see $\mathrm{SI})$.

The optical image of the sample film was initially recorded with microscope objective, as shown in Fig 2c. The FTIR spectra were extracted from the scanned area (marked by the red square in c), out of which a representative spectrum is shown in Fig. $2 d$, which exhibits the characteristics vibrational modes of the polymeric backbone of the PAni, such as asymmetric and symmetric C-C ring vibrations located at 1590 and $1504 \mathrm{~cm}^{-1}$, respectively. Besides the bands located at 1305, 1236 and $1150 \mathrm{~cm}^{-1}$ arise from the $\mathrm{N}-\mathrm{H}, \mathrm{C}-\mathrm{C}$, and in plane $\mathrm{C}-\mathrm{H}$ bending vibrations modes, respectively ${ }^{7}$. Fig. 2e shows the 2D chemical map obtained by the integration of the $\mathrm{C}-\mathrm{C}$ ring vibrational modes, highlighted in green in the FTIR spectrum. This map shows the distribution of these two bands by the colour contrast, where the blue indicates the lowest concentration and the red suggests the highest concentration ${ }^{8}$. A visual comparison with optical microscopic image confirms that the darker regions, highly concentrated with PAni content shows a similar trend in the chemical image, where these regions show the 
highest concentration (red in colour) as compared to the lighter regions, which are blue in colours. The 3D chemical map (Fig. 2f) was obtained in a similar fashion and contrast similar to 2D map was obtained. The FTIR chemical maps suggest that the deposition of the polymer occurs inhomogeneously in the form of islands in the $\mu \mathrm{m}$ range. The surface-confined electrochemical information from these conductive islands is then obtained by using FPA detector. A typical $64 \times 64$ elements FPA detector can collects 4096 spectra from the selected region of the sample with a pixel resolution of $\sim 2.5 \mu \mathrm{m}$ (see Fig. S1, SI)) in a single detection within 2 minutes, thus making possible the spatially resolved measurements in a short duration of time. This also allows the direct construction of chemical maps from the spectra collected via a mathematical treatment, which shows the chemical distribution of vibrational mode from the selected sample area, as shown previously. EVSM system was initially evaluated by performing the cyclic voltammetry to verify whether the electrodes are properly connected and that the system is suitable for carrying out the spectroelectrochemical measurements (Fig. S2, SI). Subtractively normalized interfacial infrared (SNIFTIR) spectra of the PAni film, where the spectrum recorded at each applied potential is subtracted from that obtained at the initial potential ${ }^{9}$, are initially recorded with the microscope and FPA detector in the reflectance mode. The potential window used for monitoring these spectra and hence the observed changes in the PAni electrode is the same as used in cyclic voltammetry measurements, namely -0.2 to $+0.8 \mathrm{~V}$. Fig. 3a exhibits the SNIFTIR spectra, recorded at the different applied potential. With increasing potential, some of the peaks increased in intensity, while others decreased, suggesting that the polymer chain undergoes structural changes during the potential sweep. For example, the vibrational peaks at 1576, 1307 and $1156 \mathrm{~cm}^{-1}$ gained an increase in their intensity, whereas the intensity of the absorption peaks at 1510 and 1202 $\mathrm{cm}^{-1}$ are decreased. The absorption band observed near $1500 \mathrm{~cm}^{-1}$ is assigned to the stretching vibration of the benzenoid ring of the polymer ${ }^{10}$ and the decrease in the intensity of this band suggests symmetry breaking and loss of benzenoid ring structure. On the other hand, the absorption bands observed at 1576 and 1487 

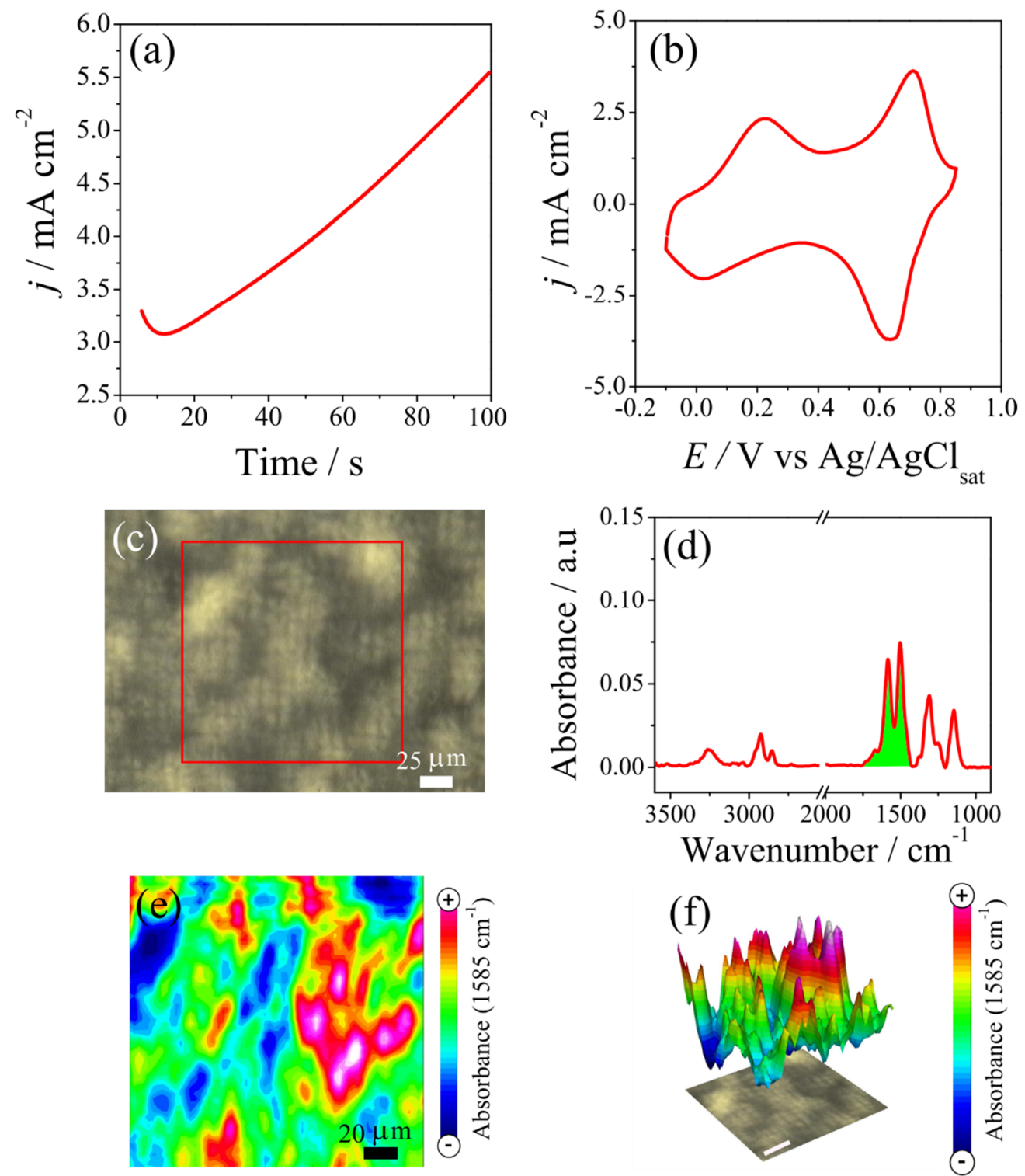

Fig. 2. Electrodeposited PAni and micro-FTIR. (a) Chronoamperometric curve of the electrodeposition of PAni on Au mirror electrode at applied potential of $0.8 \mathrm{~V}$ (aniline solution, $\left.0.1 \mathrm{~mol} \mathrm{~L}^{-1}\right)$ and $\mathrm{H}_{2} \mathrm{SO}_{4}\left(0.5 \mathrm{~mol} \mathrm{~L}^{-1}\right)$ during $100 \mathrm{~s}$. (b) Cyclic voltammogram of the resultant electrode in $\mathrm{H}_{2} \mathrm{SO}_{4}\left(0.5 \mathrm{~mol} \mathrm{~L}^{-1}\right)$. Scan rate: $50 \mathrm{mV} \mathrm{s}^{-1}$ (c) Optical microscopic image showing the PAni on a Au-coated glass substrate. The area highlighted in red is selected for the extraction of the spectra and 2D/3D chemical maps, (d) FTIR spectrum exhibiting the characteristics vibrational modes of the PAni structure, (e) 2D chemical map showing the distribution of the spectral bands highlighted by green in $d$ and (f) 3D chemical map obtained in the similar fashion as 2D. The scale bar in $\mathrm{f}$ is $40 \mu \mathrm{m}$. 
$\mathrm{cm}^{-1}$ arise from the semi-quinoid ring structure of the polymer chain ${ }^{11}$ and an increase in their intensities with the increasing potential are consistent with the conversion of benzenoid ring (i.e. (-B-NH-B-NH-B-NH-B-NH)) into semiquinoid ring or polaron lattice structure (i.e (-B-NH-B-NH $\left.{ }^{\cdot+}-\mathrm{B}-\mathrm{NH}-\mathrm{B}-\mathrm{NH}^{\bullet+}\right)$ ). Similarly, the increase in the intensity of the vibrational peaks at 1307 and 1156 $\mathrm{cm}^{-1}$ are correlated to the protonation processes of the leucomeraldine form, resulting in the conversion to the following structure: (-B-NH-B-NH-B$\mathrm{NH}^{+}=\mathrm{Q}=\mathrm{NH}^{+}$). The changes in the intensity of specific bands of the SNIFTIR spectra are followed as a function of applied potential (Fig. 3b) and are then used as reference bands for the evolution of chemical maps. All the three bands centred at 1156,1307 , and $1576 \mathrm{~cm}^{-1}$ initially gain an increase in their intensity, when the potential is increaed from $0.0 \mathrm{~V}$ to $+0.6 \mathrm{~V}$, which are consistent to the formation of semiquinoid ring and protonation processes during the conversion of reduced form (non-conducting) into partially oxidized form (conducting). In general, the strengthening in the intensity of these bands indicates an increase in the degree of doping of the polymeric backbone. Alternatively, this could be due to the increase in the dipole moment by the development of the positive charge on the polymer chain, which increases in the intensity of these absorption bands. With the further increase in the potential namely at $+0.8 \mathrm{~V}$, a decrease in the absorption intensity of these bands is observed and may be related to the loss of the conductivity of the polymer during its conversion into fully oxidized form. The appearance of the band at $1627 \mathrm{~cm}^{-1}$, which is assigned to N-ring vibration, confirm the transformation of polaron lattice into original quinoid structure ((-B$\mathrm{N}=\mathrm{Q}=\mathrm{N}-\mathrm{B}-\mathrm{N}=\mathrm{Q}=\mathrm{N}-)$ ).

Since, PAni exhibits a distinctive electrochromic behaviour, we followed the changes in colour of the PAni by recording the optical microscopic images with the increasing potential, while dealing with chemical maps and we find a trend similar to that observed in the spectra. At the initial applied potentials of - 0.2 $0.0 \mathrm{~V}$, it exists in the leucoemeraldine base form and the colour of the polymer is yellow (Fig. 3c). With the increase in the applied potential from $0.0 \mathrm{~V}-+0.6 \mathrm{~V}$, the electrode colour changes from yellow to light green and then dark green, 
corresponding the conversion into emeraldine form. As the potential exceeds the $+0.6 \mathrm{~V}$, the onset potential for the conversion to pernigraniline form, the polymer adopts a dark blue colour, as can be seen in the microscopic image recorded at $+0.8 \mathrm{~V}^{12}$

We monitor the chemical maps as a function of increasing potential, for which the areas under the vibrational peaks in the SNIFTIR spectra are integrated. Fig 3c shows the multiplex FTIR chemical images in the 2D format corresponding to two distinct vibrational modes at 1307 and $1156 \mathrm{~cm}^{-1}$. At initial applied potentials ca. $-0.2 \mathrm{~V}$ and $0.0 \mathrm{~V}$, the respective chemical maps show no contrast and are not only consistent with the spectra but with optical images at these two applied potentials, where no apparent spectroelectrochemical processes are observed. Since the electrochemical redox activity of the PAni starts only after $0.0 \mathrm{~V}$, as we have seen in the cyclic voltammogram, the first SNIFTIR spectrum with distinct vibrational modes appeared only at the $+0.2 \mathrm{~V}$. The chemical maps were obtained by integrating the bands at 1307 and $1156 \mathrm{~cm}^{-1}$, where the obtained contrast is related to the chemical distribution of these bands along the sample surface. Similar procedure was adopted at +0.4 and $+0.6 \mathrm{~V}$. The $2 \mathrm{D}$ chemical images follow a trend like the spectra for these two absorption bands. A higher concentration of these bands at the centre of the sample area, particularly in the potential interval from $0.2-0.6 \mathrm{~V}$, indicates that the polymeric molecules are not evenly distributed; instead they appear in the form of dispersed islands, when the potential on the electrode is changed. Finally, when the intensity of the absorption bands drops suddenly at $+0.8 \mathrm{~V}$, the chemical maps reveals the same effect by showing a decrease in the intensity of the polymeric islands at this higher potential. 3D chemical maps were obtained in a similar fashion, as shown in the Fig. $3 d$, where a similar tendency was observed in the colour contrast of the chemical images. All these results suggest that the electrochemical heterogeneity of the conducting polymer electrode can be successfully controlled and monitored in real time by the EVSM, showing the applicability of the technique towards the elaboration and understanding of the mechanism behind the 


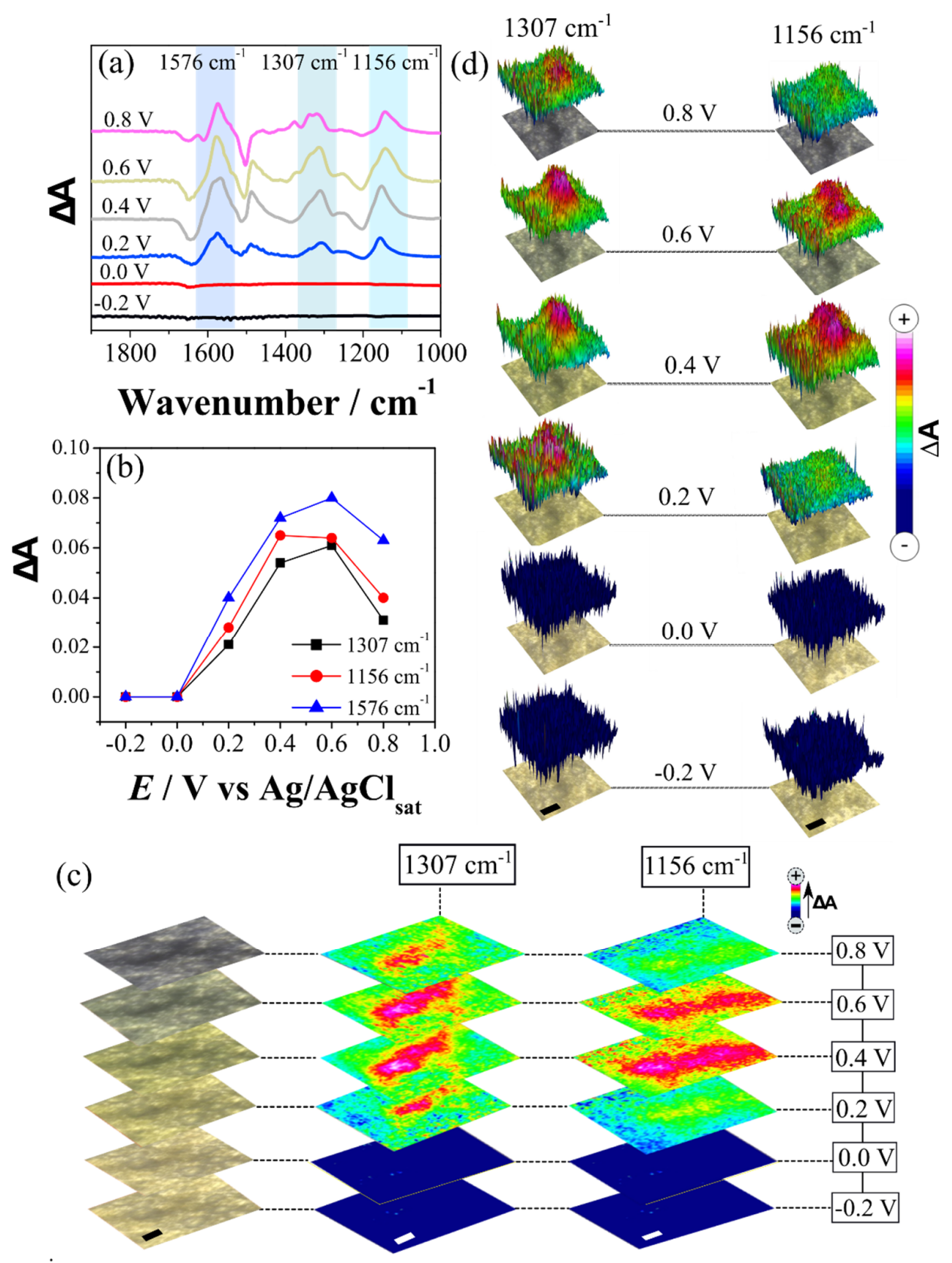

Fig. 3. EVSM of PAni modified flat Au mirror electrode recorded with FPA detector (a) Spectral changes of PAni recorded at different applied potentials in $\mathrm{H}_{2} \mathrm{SO}_{4}\left(0.5 \mathrm{~mol} \mathrm{~L}^{-1}\right)$, (b) Changes in the intensities of the spectral bands measured from SNIFTIR spectra as a function of applied potential (c) 2D chemical maps obtained by integrating the specific bands at 1307 and $1156 \mathrm{~cm}^{-1}$ from the SNIFTIR spectra shown in b, and (d) 3D chemical maps obtained in the similar fashion as in c. The scale bar is $40 \mu \mathrm{m}$. 
complex electrochemical properties changes, which can help in their proper exploitation in practical applications.

In summary, the surface morphology of PAni modified flat Au mirror electrode was evaluated by using micro-FTIR spectroscopy and then underwent through in-situ monitoring of the surface redox reaction in real time through micro-FTIR spectroscopy coupled to the electrochemistry. Consistent results between the changes in the vibrational modes in the SNIFTIR spectra and the consequent 2D and 3D FTIR chemical maps were obtained at different applied potential. The results obtained are promising in the sense, that regardless of the restrictions imposed by the experimental conditions, such as the interference of absorption mode of the water (OH bending), in the region of $1590 \mathrm{~cm}^{-1}$, the changes in the spectral properties of the polymeric islands are successfully monitored, which gave an insight into their topological disorder along the entire electrode area and may pave the way for the better understanding of the charge transfer hindrance due to poor chain connectivity.

The authors gratefully acknowledge the São Paulo Research Foundation (FAPESP) for the financial support of the research projects under the grant numbers: (2019/15333-1, 2019/12053-8, 2017/20493-2), and Coordinating Agency for Advanced Training of Graduate Personnel (CAPES) grant number: (88887.358060/2019-00). The financial support from National Council of Scientific and Technological Development (CNPq) is also acknowledged.

\section{Conflicts of interest}

There are no conflicts to declare.

\section{References}

1 M. B. Regasa, T. R. Soreta, O. E. Femi, P. C. Ramamurthy and S. Kumar, J. Mol. Recognit., 2020, 1-11.

2 T. Zhang, H. Qi, Z. Liao, Y. D. Horev, L. A. Panes-Ruiz, P. S. Petkov, Z. Zhang, R. Shivhare, P. Zhang, K. Liu, V. Bezugly, S. Liu, Z. Zheng, S. Mannsfeld, T. Heine, G. Cuniberti, H. Haick, E. Zschech, U. Kaiser, R. Dong and X. Feng, Nat. Commun., 
2019, 10, 1-9.

3 P. Kumar, P. Verma, R. Singh and R. K. Patel, in Proceeding of International Conference on Intelligent Communication, 2016, pp. 979-989.

$4 \quad$ Langmuir, 2020, 36, 4153-4164.

$5 \quad$ BR 102019001293 5, 2019.

6 L. J. A. Macedo and F. N. Crespilho, Anal. Chem., 2018, 90, 1487-1491.

$7 \quad$ M. Tanzifi, S. H. Hosseini, A. D. Kiadehi, M. Olazar, K. Karimipour, R. Rezaiemehr and I. Ali, J. Mol. Liq., 2017, 244, 189-200.

8 L. J. A. Macedo, F. C. D. A. Lima, R. G. Amorim, R. O. Freitas, A. Yadav, R. M. lost, K. Balasubramanian and F. N. Crespilho, Nanoscale, 2018, 10, 15048-15057.

9 Z. Su, V. Climent, J. Leitch, V. Zamlynny, J. M. Feliu and J. Lipkowski, Phys. Chem. Chem. Phys., 2010, 12, 15231-15239.

10 M. Trchová and J. Stejskal, Pure Appl. Chem., 2011, 83, 1803-1817.

11 G. A. Planes, J. L. Rodríguez, M. C. Miras, G. García, E. Pastor and C. A. Barbero, Phys. Chem. Chem. Phys., 2010, 12, 10584-10593.

12 Z. Shi, Y. Li, X. Chen, H. Han and G. Yang, Nanoscale, 2014, 6, 970-977. 
Electronic Supplementary information

Electrochemical-Coupled Vibrational Spectromicroscopy Recognize Conductive Islands in Topological Redox Surface

Ayaz Hassan, Lucyano J. A. Macedo, Frank N. Crespilho*

São Carlo Institute of Chemistry, University of São Paulo, São Carlos SP, 13560-970, Brazil

Corresponding author: frankcrespilho@iqsc.usp.br 


\section{Electrodeposition of the PAni film}

A thin film of $\mathrm{Au}(\sim 20 \mathrm{~nm})$ was obtained through vacuum evaporation on glass substrate, previously evaporated with $\mathrm{Ti}(\sim 10 \mathrm{~nm})$ as adhesion layer. The resultant Au-coated glass substrate, from here onward named as flat Au mirror electrode, was used as working electrode and modified with the PANI film by applying a potential of $0.8 \mathrm{~V} \mathrm{vs} \mathrm{Ag} / \mathrm{AgClsat}$ electrode in a solution containing a freshly distilled aniline $\left(0.1 \mathrm{~mol} \mathrm{~L}^{-1}\right)$ and $\mathrm{H}_{2} \mathrm{SO}_{4}(0.5$ $\mathrm{mol} \mathrm{L}^{-1}$ ) for $100 \mathrm{~s}$. A one compartment, three electrodes electrochemical cell controlled by the potentiostat/galavanostat (Autolab, PGSTAT128N) was used for this purpose, consisting of the flat Au mirror as the working electrode, Pt wire counter electrode, and $\mathrm{Ag} / \mathrm{AgCl}_{\text {sat }}$ reference electrode. All the potentials are quoted against $\mathrm{Ag} / \mathrm{AgCl}$ sat.

\section{Electrochemical assembly}

The spectroelectrochemical measurements were performed in a home-built polytetrafluoroethylene (PTFE) three electrodes electrochemical cell containing the PAni modified flat Au mirror as the working electrode, Pt wire sealed in a glass tube as counter electrode and $\mathrm{Ag} / \mathrm{AgCl}$ sat as reference electrode. The electrochemical cell was connected to the potentiostat/galvanostat (autolab, PGSTAT128N), where the potential of the working electrode was maintained at fixed value through the technique of steadystate staircase voltammetry for a fixed duration of time, before switching to the next potential.

\section{Electrochemical coupled vibrational spectromicroscopy (EVSM) setup}

The spectroelectrochemical cell was mounted in the motorized stage of the FTIR microscope (Hyperion 3000) coupled to FTIR spectrometer (Vertex 70v, Bruker), which provides the facility of moving the electrochemical cell and hence the measurement can be performed at any desired position of the working electrode by initially focusing the visible and then IR light through $\mathrm{CaF}_{2}$ window, when the measurement is performed. The $\mathrm{CaF}_{2}$ window also controls the thickness of the electrolyte layer above the working electrode. A gasket made of PVC was placed between the $\mathrm{CaF}_{2}$ window and the surface of the working electrode. The FTIR spectra were recorded with the FPA detector at each applied potential ranging from $-0.2 \mathrm{~V}$ to $+0.8 \mathrm{~V}$ and the FTIR chemical maps were then obtained through the integration of the specific spectral bands at particular applied 
potential. The reference spectrum was recorded initially during the application of the $0.2 \mathrm{~V}$ potential to the working electrode and used for consequent measurements of the spectra and chemical maps. The resultant spectra are called as substractively normalized interfacial FTIR (SNIFTIR) spectra. These spectra were collected in the spectral range from $4000-900 \mathrm{~cm}^{-1}$ at a spectral resolution of $8 \mathrm{~cm}^{-1}$ with the 32 scans co-added. 
S1: Spatial resolution of the electrochemical coupled vibrational spectromicroscopy (EVSM)
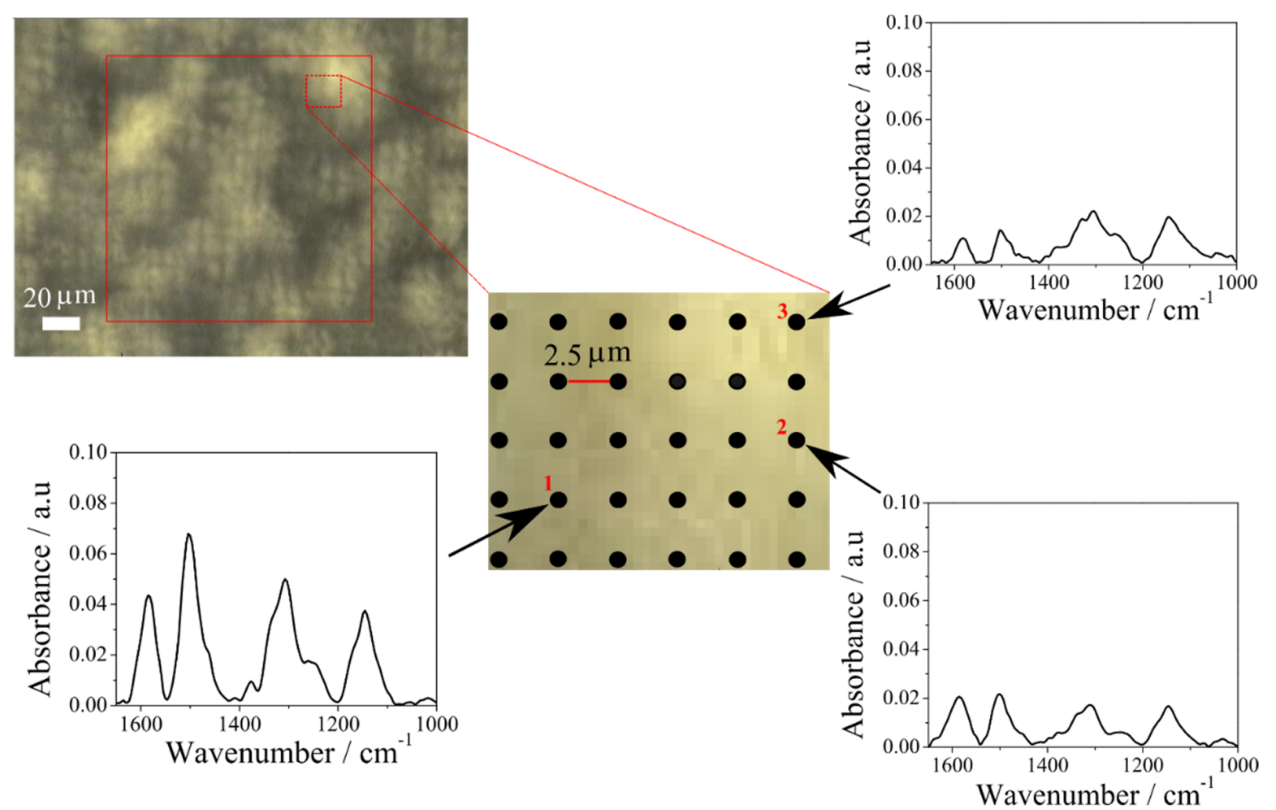

Fig. S1: Illustration of the spatial distribution of the electrochemical coupled vibrational spectromicroscopy technique: A sub-scanned area of the electrode partly covered with polymeric molecules is selected from the optical microscopic image and evaluated for spatial distribution. The black dots correspond to the individual pixels, each composed of single spectrum and the distance between them is $2.5 \mu \mathrm{m}$. Spectra are extracted from three different regions of the microscopic image and each spectrum shows a different level of intensity of the peaks depending on the region from where it is taken. The spectrum from region 1, which is apparently darker and contain larger contents of the polymeric materials show the highest intensity as compared to region 3 , containing relatively smaller contents. The spectrum from region 2 shows the intermediate intensity. 
S2: Testing of the EVSM system by performing the cyclic voltammetry

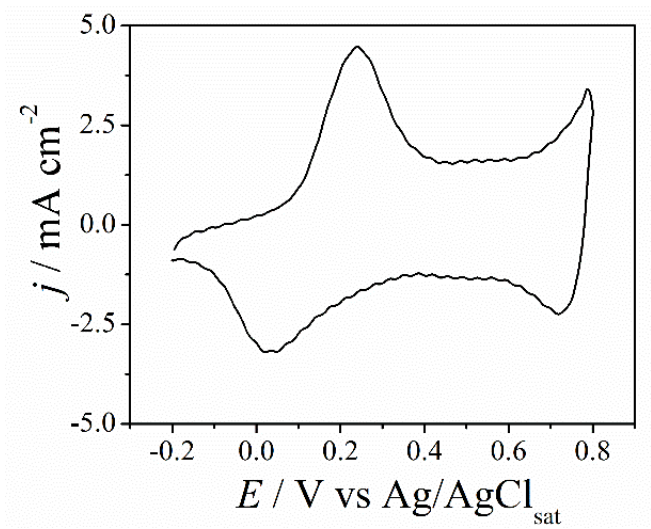

Fig. S2: Cyclic voltammogram of the PAni modified Au mirror electrode performed in the EVSM setup in $\mathrm{H}_{2} \mathrm{SO}_{4}\left(0.5 \mathrm{~mol} \mathrm{~L}^{-1}\right)$ in the potential range of -0.2 to $+0.8 \mathrm{~V}$. Scan rate $=50$ $\mathrm{mV} \mathrm{s}^{-1}$ 